PHYSICAL REVIEW B 83, 159901 (2011)

\title{
Publisher's Note: Intra-Landau-level magnetoexcitons and the transition between quantum Hall states in undoped bilayer graphene [Phys. Rev. B 83, 115455 (2011)]
}

Csaba Tőke and Vladimir I. Fal'ko

(Received 1 April 2011; published 12 April 2011)

DOI: 10.1103/PhysRevB.83.159901

PACS number(s): 72.80.Vp, 71.35.Ji, 71.70.Di, 73.43.Lp, 99.10.Fg

This paper was published online on 29 March 2011 without the author's corrections incorporated into the published article. The paper has been corrected as of 4 April 2011. The text is correct in the printed version of the journal. 